\title{
NEONATAL DIARRHOEA DUE TO SALMONELLA PARATYPHI B
}

\author{
BY \\ D. M. MARTYN JONES AND C. G. PANTIN \\ From the Department of Public Health and the Clegg Memorial Laboratory, Isle of Man
}

(RECEIVED FOR PUBLICATION JULY 28, 1955)

This is an account of a small outbreak of neonatal diarrhoea in a maternity home.

\section{History of Outbreak}

The infection was introduced into the maternity home by a Mrs. C., who had a bout of diarrhoea and vomiting four days before she was admitted on May 27, her membranes having ruptured prematurely. She was delivered in her own single room within an hour of admission, so was in effect isolated from the outset. As Mrs. C. reported neither her original attack nor the diarrhoea which was continuing her baby was placed in the communal nursery.

On June 1, four days later, Baby C. and Baby H., who had been born only 36 hours before, were taken ill. The following day two more infants, Baby $K$. and Baby S., were ill. All four infants were removed to isolation quarters on June 2.

On June 3 the maternity home was closed to further admissions, and by the evening of June 4 the remaining 18 infants had been sent home with their mothers. These infants and the six sent home during the previous week were apparently healthy.

\section{Incubation}

Baby K.'s history established that infection was spreading in the nursery after the early hours of May 31, when he was born. The three babies had an incubation period of 24 to 36 hours.

The four infants had a pyrexia with loss of weight and appetite, together with diarrhoea, the stools being green, often slimy, and sometimes blood-stained. Baby C. died. Babies H., K., and S. recovered, being given chloromycetin and fluids subcutaneously or by stomach tube.

A rectal swab was taken from Mrs. C. on May 29 from which $S$. paratyphi B was isolated. $S$. para. typhi B was recovered from the bowel of all four babies, and in addition from the heart blood of Baby C.

The organism recovered from the intracardiac blood was sent to the Salmonella Reference Laboratory and was reported on as follows :

"This organism has the following antigenic structure : $\mathrm{O}=4,5,12 ; \mathrm{H}=\mathrm{b}-1,2$. Therefore it is Salmonella paratyphi B."
The organisms recovered from the first seven cases were sent for phage typing to the Central Enteric Reference Laboratory and Bureau, Colindale, and were reported as being untypable.

\section{Investigation of Contacts}

Arrangements were made to take rectal swabs from 90 contacts. Three such swabs were taken at two to three days' interval from 18 members of the maternity home staff, including the boiler-man. All were negative.

All the 28 mothers who were in the maternity home during the week May 27 to June 3 submitted rectal swabs. One besides Mrs. C. was found to be excreting $S$. paratyphi $\mathrm{B}$, and she was nursing an infected infant.

Of the 24 infants sent home all were swabbed and nine were found to be excreting $S$. paratyphi B ; the contacts of these nine were swabbed. By the time the infected infants had been collected into the Isolation Hospital two adults, the father and a grandmother of one infant, and three children, siblings of two other infants, were infected.

Later, a mother was infected by the infant she was nursing in the Isolation Hospital.

Fig. 1 shows the spread and extent of the epidemic.

\section{Discussion}

Mrs. C. presumably infected her baby at birth. How the infection from Baby $C$. spread in the nursery is not certain, but contamination of the feeding-bottles seemed a possibility. All infants were given drinks by bottle on the first day after birth. Six infants continued to be bottle-fed after this time, and of these five were infected. Fourteen infants were breast-fed, and only four of these were infected.

Among those infected few were ill. The symptoms of the four babies recognized as ill in the maternity home have been described. Of the nine babies found to be excreting $S$. paratyphi B after they had returned home five had green stools and were off colour and of poor appetite and four had no symptoms. These thrived and continued to 


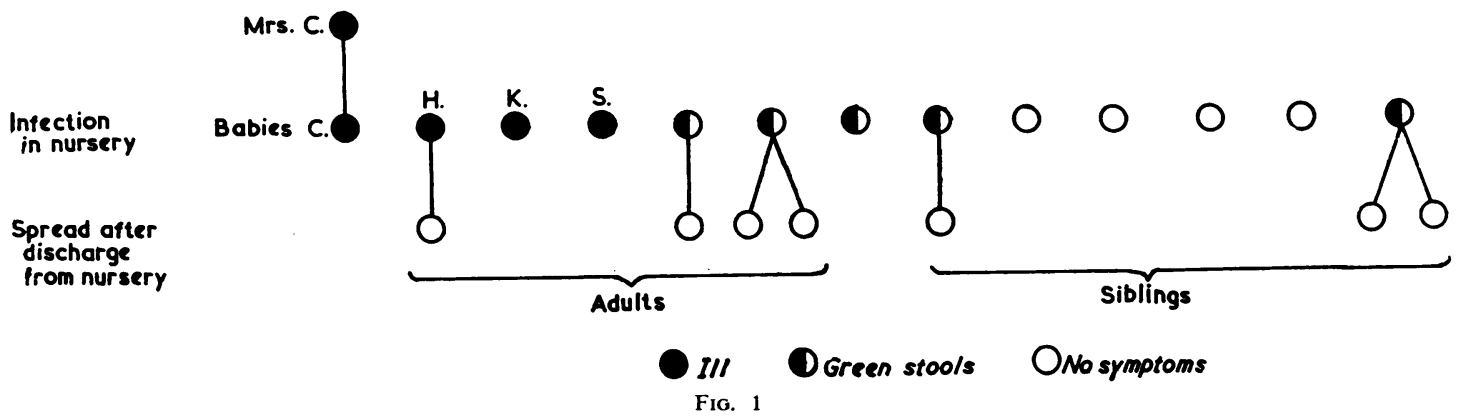

excrete $S$. paratyphi B for months without any evidence of ill-health.

Of the siblings infected, two had no symptoms, while the third, a girl of $2 \frac{1}{2}$ years, was very sick. She had been in contact with her infected baby brother for nine days before she developed diarrhoea. She was removed at once to the Isolation Hospital. Her temperature was $101.6^{\circ} \mathrm{F}$., and she was so ill that she was incontinent of faeces and urine. Her clinical condition improved rapidly after chloramphenicol had been given. In her case only enteric fever might have been suspected on clinical grounds.

None of the infected adults was ill. One alone showed a significant rise in titre, from 1 in 20 to 1 in 80, against $S$. paratyphi $\mathrm{B} \mathrm{H}$ and $\mathrm{O}$ antigens.

From two of the adults $S$. paratyphi B was only recovered over a period of a few days. One of these two was found serving ice-cream to a party of 300 guests, but no case resulted.

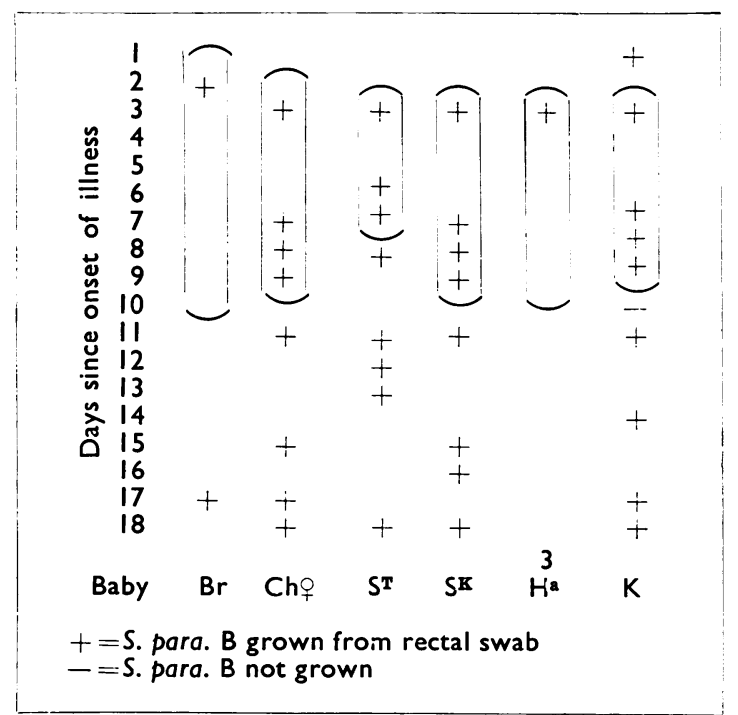

FIG. 2.-Six infants given chloramphenicol 0.125 g. $\times 4$ daily for the number of days indicated by the "lozenge."
Six of the 12 surviving infants and the $2 \frac{1}{2}$-yearold girl were sufficiently ill to be given chloromycetin. They responded well clinically, but the treatment had no effect on the excretion of $S$. paratyphi B, as Fig. 2 shows.

One infant $\left(\mathrm{H}^{\mathrm{a}}\right)$ was never again found to be excreting $S$. paratyphi $\mathrm{B}$, but the number of rectal swabs taken over the relevant period is inadequate. This was the only infant treated at home.

The outbreak then left us with a number of carriers.

\section{Method}

Each rectal swab was seeded on to one plate of desoxycholate agar and on to one of Wilson and Blair's medium. Then, the stem of the swab being of wood, the cotton-wool head was broken off and left in a bottle of "selenite." The desoxycholate plate was examined after 24 hours' incubation and, if $S$. paratyphi B was not found, a further desoxycholate plate was inoculated from the "selenite F." This plate and the original Wilson and Blair plate were examined after a further 24 hours had elapsed.

All the media were obtained from Dr. McCartney's laboratory.

Those taking the swabs were not always very skilled.

\section{Bacteriological Results}

During a period when the 12 infected infants were excreting $S$. paratyphi $\mathrm{B}$, that is, during a period covered in each infant from the first positive swab to the last positive swab, we examined 323 rectal swabs by this method and recovered $S$. paratyphi B from 259 of them, that is, from $80 \%$.

We found that at first $S$. paratyphi B grew freely on the primary desoxycholate plate, then they became scanty, and finally $S$. paratyphi B was only recovered after enrichment in "selenite F."

When this stage was reached in one infant we began to culture from rectal swabs and faeces in parallel. The amount of faeces that was put in the "selenite" was about the size of a pea. Once $S$. paratyphi B was recovered from both swabs and 
faeces ; four times it grew from the swab alone ; not once did we get growth from the faeces and not from the rectal swab.

Our acceptance of six consecutive negative rectal swabs as indicating the end of the carrier state seems, therefore, to be rational.

However, of 11 carriers on whom we continued to examine rectal swabs after they had had six consecutive negative results, two had further positive swabs. Of these the first was one of twins and probably had cleared up but had been reinfected by its twin. These twins belonged to an unhygienic family, five of six members having been found to be excreting $S$. paratyphi B. From the second infant $S$. paratyphi B was recovered on June 9. It was thriving. Seven consecutive negative swabs followed. Then on July 19 the infant became ill with diarrhoea and $S$. paratyphi B reappeared. Chloromycetin was given, and although the baby improved clinically $S$. paratyphi B was recovered every time from six consecutive swabs. Terramycin was given. Six negative swabs followed and then once again the infant relapsed with enteritis and again $S$. paratyphi $B$ was recovered from the swabs. The first relapse occurred when other infected infants were in the Isolation Hospital; the second relapse occurred when it was the only remaining infant.

With this type of carrier one can never be sure that he is clear, and he might be described as an " intermittent excreter."

Two of the adults excreted $S$. paratyphi B for less than a week and all had ceased to excrete by 10 weeks.

One infant excreted $S$. paratyphi B for not more than three weeks. The behaviour of two, the twin and the intermittent excreter, has already been described. The remaining nine infants went on for months, with $S$. paratyphi B recovered from practically every swab in gradually decreasing numbers, till in some cases the organism abruptly ceased to be recovered. Others were still excreting nine months after infection.

Of all 12 surviving infants six were still excreting after six months and three after nine months.

At one stage an attempt was made to end the carrier state in six infants with terramycin. Fig. 3 shows the result.

For one day all gave negative swabs, but soon $S$. paratyphi B was recovered from the rectal swabs of four of them. Another, the so-called intermittent excreter, relapsed after a few weeks. One infant cleared completely after terramycin.

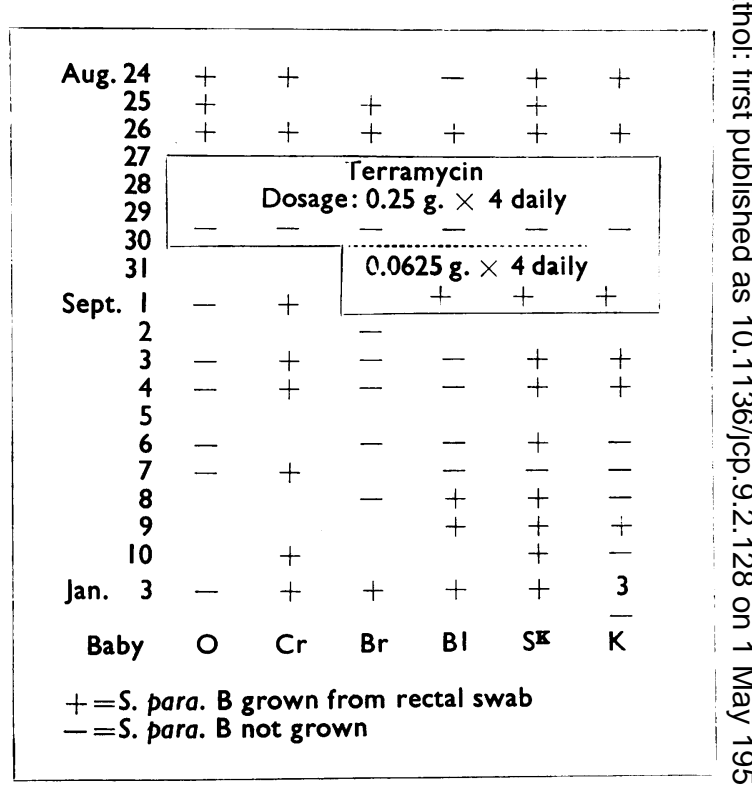

FIG. 3.-Effect of terramycin on the recovery of $S$. para. B from $S$ the rectal swabs of six infant carriers.

\section{Summary}

An outbreak of neonatal diarrhoea due to Salmonella paratyphi $\mathrm{B}$ is described.

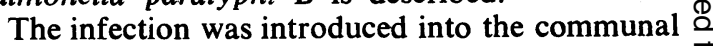
nursery by a newborn infant who had been infected $\overrightarrow{\overrightarrow{0}}$ at birth by the mother. In the course of a week 3 this infant and three others became ill and the maternity home was closed.

From the 24 infant contacts sent home as fit, 응 $S$. paratyphi B was isolated from nine. Of these, five appeared to have been off colour or had green 3 . stools, but four were unaffected by the infection.

Four adults and three siblings, contacts of in- 3 fected babies, became infected.

The infants remained carriers for longer than the adul's or older children. Three were still $\frac{D}{0}$ excreting $S$. paratyphi B nine months after infection.

Chloromycetin appeared to be of value in com- $N$ bating the symptoms, but had no effect in the N carrier state.

Terramycin appeared slightly more effective in terminating the period of excretion, but five out of $\stackrel{\circ}{\bar{C}}$ six infants treated soon reverted to the carrier $\stackrel{\mathbb{Q}}{\rightarrow}$ state.

Our thanks are due to Dr. Joan Taylor for confirming that the organism involved was indeed $S$. para- $\stackrel{\mathbb{Q}}{?}$ typhi B, and to Dr. E. S. Anderson for showing that $\underset{\mathbb{Q}}{\mathbb{Q}}$ the organism belonged to an untypable strain. 